\title{
Bladder carcinoma in a 31-year-old female spina bifida patient with an auto-augmented bladder
}

\author{
Paul W. Veenboer · Laetitia M. O. de Kort
}

Received: 21 June 2011/Accepted: 3 August 2011/Published online: 26 August 2011

(C) The Author(s) 2011. This article is published with open access at Springerlink.com

\begin{abstract}
We present the case of a 31-year-old spina bifida patient presenting with a poorly differentiated T3N1M0 bladder carcinoma with sarcomatoid differentiation in an auto-augmented bladder. She underwent a radical cystectomy and a bilateral lymph node dissection. However, only 10 months after the onset of her symptoms, she died after major lymphatic metastases had developed in the small pelvis. This case report is the first on an adult spina bifida patient developing bladder carcinoma after detrusorectomy. It shows that bladder cancer also occurs in patients who underwent detrusorectomy, despite the fact that the risk is supposedly lower than in patients who underwent enterocystoplasty. Moreover, tumour spread to adjacent organs could occur more rapidly in auto-augmented bladders because of the lack of muscle tissue. The latter could have serious implications on the prognosis of these patients.
\end{abstract}

Keywords Bladder carcinoma $\cdot$ Bladder malignancy $\cdot$ Myelomeningocele $\cdot$ Spina bifida . Spinal dysraphism

P. W. Veenboer $(\bowtie) \cdot$ L. M. O. de Kort

Department of Urology, University Medical Centre Utrecht (UMC Utrecht), Heidelberglaan 100, Huispostnr. C.04.236, 3584 CX Utrecht, The Netherlands

e-mail: p.w.veenboer@students.uu.nl

\section{Introduction}

As spina bifida patients (SB patients) reach adulthood more often nowadays, clinicians are confronted with new problems that were not encountered in the past. SB patients are reported to develop bladder cancer more often and earlier than their healthy peers. Several case reports present SB patients with highgrade, locally advanced bladder carcinoma at relatively young age. We would like to report on the case of a 31-year-old female SB patient who developed bladder carcinoma in an auto-augmented bladder.

\section{Case report}

A 31-year-old female spina bifida patient presented at our outpatient clinic in the autumn of 2008. She was known with a lumbar myelomeningocele (level L3) with primary closure in early childhood. In 1992, she had bladder stones removed by transurethral cystolithotripsy. In 1994, she underwent a detrusorectomy (auto-augmentation; enlargement of bladder capacity by removal of the muscle layer), rectus-fascia-sling operation and Mitrofanoff procedure (appendicovesicostomy; the creation of an umbilical stoma to facilitate easier self-catheterisation). Now, she presented with complaints of vague aching in the lower abdomen. She also complained of foul-smelling, cloudy urine. She did not smoke and used only Metamucil ${ }^{\circledR}$ because of constipation. Physical examination 
revealed no specific signs. Urinalysis showed a leukocyturia [500/High Power Field (HPF)] and erythrocyturia (30 erythrocytes/HPF). Urine culture showed a mixed flora Streptococcus, Bacteroides and Peptostreptococcus species. Ultrasound of the bladder showed a calcification of the bladder wall. Computed tomography-intravenous pyelography (CT-IVP) demonstrated a markedly thickened posterior bladder wall. Urethrocystoscopy was extremely painful. A red, irritated bladder mucosa with oedema and debris was seen, with a few small bladder stones. Since this investigation was considered not reliable, a second urethrocystoscopy under general anaesthesia was done a few weeks later. An ulcerating tumour was seen, of which a biopsy was taken. Histological examination revealed a poorly differentiated transitional cell carcinoma with muscle-invasive growth. A subsequent MAG3 renography revealed an impaired drainage of the right kidney and a diminished renal split function on the right side ( $\mathrm{L}: \mathrm{R}=64 \%: 34 \%$ ). On a plain $\mathrm{CT}$ abdomen, hydronephrosis and an obstructing mass at the distal ureter on the right side were seen (Fig. 1). Besides, para-iliac lymphadenopathy and induration of perivesical fat were detected. No further clues for metastasis were seen on chest X-ray and CT thorax. A nephrostomy tube was inserted to relieve the right kidney in early December. In mid-December, she underwent a radical cystectomy and pelvic lymph node dissection with subsequent Bricker deviation.

At histological examination, a muscle and blood vessel invasive, poorly differentiated transitional cell carcinoma with sarcomatoid differentiation was seen.

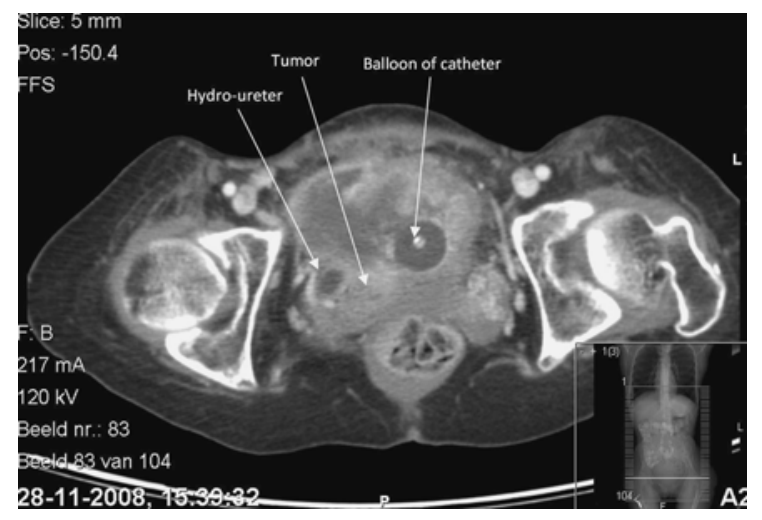

Fig. 1 Computed tomography of the lower abdomen after biopsy had revealed bladder carcinoma. Note the marked irregular aspect of the bladder wall, the distal hydroureter at the right side near the ureterovesical junction

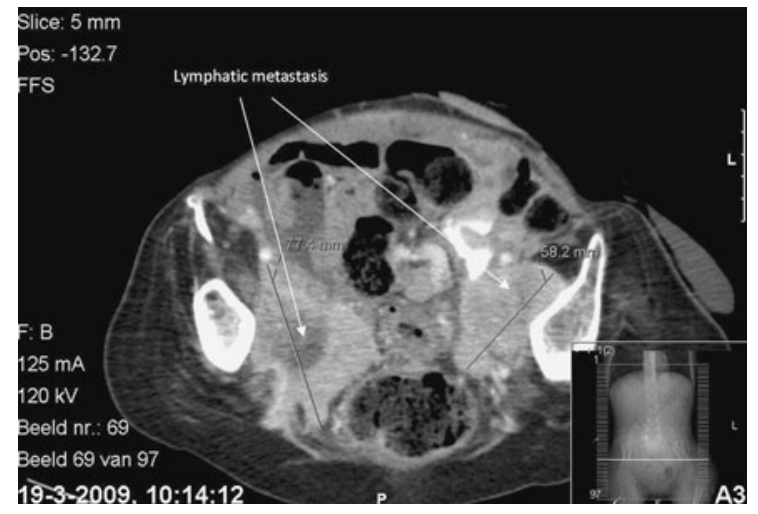

Fig. 2 Computed tomography-intravenous pyelography (CTIVP) at the time of metastasis. Two lymph node metastases (77.4 and $58.2 \mathrm{~mm})$ in the small pelvis are clearly visible

The tumour measured 10 centimetres with a maximal infiltration depth of 1.8 centimetres and was removed radically. One of the para-iliac lymph nodes contained a metastasis of the bladder tumour. Eventually, the TNM tumour stadium was T3N1M0 (TNM Classification 2002), grade III (WHO 1973), with sarcomatoid differentiation. Three months later, at CT abdomen, large bilateral lymph node masses were detected, very suggestive for metastasis (Fig. 2). Also, infiltration to the sigmoid colon was seen, as infiltration to the iliac veins. She was referred to medical oncology but found to be unfit for systemic chemotherapy because of a body weight of 36 kilograms and a poor nutritional status. She was a candidate for undergoing stenting of the iliac veins, since pain and oedema developed in the lower extremities due to venous drainage obstruction in the small pelvis. However, this was eventually not done because she developed urosepsis at the day of operation. Constipation developed because of a mass effect of the metastases on the sigmoid colon. The consulting surgeon decided to treat this conservatively. She was referred to a hospice with no further therapy than adequate analgesia and comforting and died in June 2009 at the age of 32 years, about 8 months after diagnosis.

\section{Discussion}

We present the case of a 31-year-old female SB patient with T3N1M0 poorly differentiated transitional cell bladder carcinoma with sarcomatoid 
differentiation. She was relatively young, presented with lymph node metastasis and muscle-invasive growth and presented atypically with lower abdominal pain and urinary tract infection. After performing a literature search, we could only retrieve one other case report of the same histological type of bladder cancer with spina bifida (a 32 year-old SB patient with a grade III T3 tumour with sarcomatoid differentiation, who was successfully treated by radical cystectomy and who is free of disease at 9 months of follow-up) [1].

Proposed risk factors of developing bladder malignancy in SB patients are chronic urinary tract infection, stone disease and catheterisation and surgery incorporating enteric segments into the lower urinary tract like enterocystoplasty [2]. The latter procedure is often performed in patients with a low functional bladder capacity. Although functional outcomes are reported to be good, enterocystoplasty can give rise to complications like metabolic anomalies, urolithiasis and even bladder carcinoma, since urine is carcinogenic for the enteric mucosa. The latter issue has also been recognised before in other urine-storing reservoirs (pouches, neobladders). Detrusorectomy has been developed in the late 1980s to offer a less invasive alternative for enterocystoplasty [3]. One of the possible advantages could be the lower risk of developing bladder carcinoma. Detrusorectomy is not a known risk factor and has only been reported once before (in a 20-year-old patient who had pT2N2Mx disease and eventually died 15 months after onset of symptoms) [2]. It is important to realise that bladder carcinoma not only develops in patients who underwent augmentation using enteric segments.

In an auto-augmented bladder, staging of the primary bladder tumour might pose a challenge because of the absence of muscle in the bladder wall. The term 'muscle-invasive' does not apply anymore, and tumours arising in the auto-augmented part of the bladder cannot be staged as T2. Instead, every tumour beyond stage $\mathrm{T} 1$ is automatically graded T3 or T4. The difficulty of staging has been recognised before in elderly patients with bladder carcinoma in bladder diverticula. In this subset of patients, rapid invasion of perivesical tissues has been observed [4]. It seems that the same concepts could be applied in patients who underwent a detrusorectomy, since a detrusorectomy is essentially nothing more than creating a large pseudo-diverticulum on the bladder roof. Taking this into account, bladder carcinoma arising in the augmented part of the bladder carries a poor prognosis. In the patient described in this case report, in biopsy seen after transurethral resection, muscle was present (the biopsy was taken next to the ureteral ostia), but the tumour was very large at presentation and had already grown into the pseudo-diverticulum.

Although SB patients are theoretically more prone to develop bladder malignancy, the number of case reports on this subject is still relatively limited. After reviewing our own database of 260 adult SB patients who have been in follow-up in our own centre in the past decade, only one patient $(0.4 \%)$ with bladder cancer was found (which is the one presented here). Therefore, the exact incidence of bladder cancer in SB patients remains uncertain.

One earlier series of Pagès et al. [5] presents four SB patients with bladder cancer with a mean age of 32.75 years. All these patients had undergone enterocystoplasty, and 3 of them performed clean intermittent catheterisation. All had a history of recurrent urinary tract infections. Presentation varied; only one patient presented with 'classical' painless haematuria, two with abdominal pain and one with ureteral obstruction. Three out of four patients presented with locally advanced disease (T3) or lymph node metastasis at the time of presentation. Austin et al. [6] presented a series of 8 patients with a mean age of 41 years. Again, a history of chronic infection was present in all 8 patients; only 1 out of 8 patients had a history of smoking. Only one patient had undergone bladder augmentation in the past. Patients presented with either gross haematuria $(n=5)$ or renal failure due to ureteral obstruction $(n=2)$. Seven of these patients had a locally advanced tumour or lymph node involvement at the time of presentation. Median survival was 6 months in these patients. Subsequently, they added an additional 11 patients at their series which were previously published in the literature (the series of Austin provides a review of all case reports until 2007). Median survival (if available) was 6 months, with locally advanced (T3) or lymph nodal involvement at the time of presentation in 15 out of 16 patients with staging data available. The concepts of increased risk of developing bladder carcinoma are also (or at least in part) applicable to patients suffering from other neurological conditions 
causing neurogenic bladder, since they are exposed to the same risk factors. A 2007 series from Parra et al. [7] presented 8 patients with spina cord injuries $(n=4)$, spina bifida $(n=1)$ familial spastic tetraplegia $(n=1)$ and idiopathic peripheral syndrome $(n=1)$ with bladder cancer. Seven out of eight tumours were high grade, and the same amount was muscle-invasive (T2+). After a follow-up of 14-71 months, 3 patients died eventually. Thus, this series reflects that malignant degeneration of the bladder does not only occur in spina bifida patients but may occur in all patients with neurogenic bladder disorder.

\section{Conclusion}

Bladder cancer in adult spina bifida patients may arise at an early age and may present atypically, as was demonstrated by our case. It is important that clinicians are aware of the possibility and the biological behaviour of bladder cancer in this specific subset of patients. This is the first case of bladder carcinoma arising in an auto-augmented bladder in a spina bifida patient. Bladder carcinoma in an autoaugmented bladder spreads more easily to perivesical tissues because of the absent muscle layer.

Conflict of interest The authors state that they have no conflict of interest to report.
Open Access This article is distributed under the terms of the Creative Commons Attribution Noncommercial License which permits any noncommercial use, distribution, and reproduction in any medium, provided the original author(s) and source are credited.

\section{References}

1. Nomikos M, Philippou P, Glava C, Delakas D (2009) Urothelial carcinoma of the sarcomatoid variant in a young patient with spina bifida: a case report and review of the literature. Cases J 22:9381

2. Mehan R, Misseri R, Vanderbrink B, Rink RC, Beck SDW (2011) Metastatic transitional cell carcinoma of the bladder arising in a patient with bladder auto-augmentation. J Pediatr Urol 7:137-139

3. Cartwright PC, Snow BW (1989) Bladder augmentation: partial detrusor excision to augment the bladder without use of bowel. J Urol 142:1050

4. Haecker A, Riedasch G, Langbein S, Alken P, Michel MS (2005) Diverticular carcinoma of the urinary bladder: diagnosis and treatment problems. A case report. Med Princ Pract 14:121-124

5. Pagès E, Montesinos L, Meléndez M, Rodríguez S, Cuxart A (2010) Patients with spina bifida and bladder cancer. Our experience. Cerebrospinal Fluid Res 7(suppl 1):54

6. Austin JC, Elliott S, Cooper CS (2007) Patients with spina bifida and bladder cancer: atypical presentation, advanced stage and poor survival. J Urol 178:798-801

7. Parra J, Drouin S, Comperat E, Misraï V, Van Glabeke E, Richard F et al (2007) Bladder cancer in neurological patients: analysis of a single-centre series. Prog Urol 17: 1333-1336 